\title{
A cadaver pilot study to evaluate the impact of the needle bevel orientation on the ease of paravertebral catheter insertion
}

\author{
Maxim Roy, MD (D) Florian Robin, MD • Detlev Grabs, MD, PhD • \\ Laurent Letourneau-Guillon, MD, MSc, FRCPC • Monique Ruel, RN, CCRP • \\ François Girard, MD, FRCPC · Sébastien Garneau, MD, FRCPC
}

Received: 8 May 2019/Revised: 8 August 2019/Accepted: 8 August 2019/Published online: 26 August 2019

(C) Canadian Anesthesiologists' Society 2019

\section{To the Editor,}

The paravertebral block is a well-established analgesic modality, but a single-injection technique is limited to a maximum effect of approximately ten hours. ${ }^{1}$ To prolong analgesia beyond that, a continuous catheter-based technique is favoured. ${ }^{2}$ Nevertheless, a cadaver study has shown that up to $30 \%$ of catheters are impossible to insert. ${ }^{3}$ Even after successful insertion of the catheter, its migration in the paravertebral space is often unpredictable, resulting in an additional $33 \%$ incidence of misplacement. ${ }^{4}$ When a longitudinal parasagittal in-plane approach is used with the bevel oriented in a cephalad direction (as in our centre), we believe that the insertion of the catheter could be either partially or entirely impeded by the "rib-transverse process" osseous complex, which forms an incomplete anterior boundary of each paravertebral level and thus would be in close contact with the needle tip. ${ }^{5}$ The

M. Roy, MD ( $) \cdot$ M. Ruel, RN, CCRP .

F. Girard, MD, FRCPC - S. Garneau, MD, FRCPC

Department of Anesthesiology, Centre Hospitalier de

l'Université de Montréal (CHUM), Montreal, QC, Canada

e-mail: maxim.roy20@gmail.com

F. Robin, MD

Department of Anesthesiology, Centre Hospitalier de

l'Université de Montréal (CHUM), Montreal, QC, Canada

Department of Anesthesiology, Centre Hospitalier Universitaire Pellegrin, Bordeaux, France

D. Grabs, MD, PhD

Department of Anatomy, Université du Québec à Trois-Rivières (UQTR), Trois-Rivières, QC, Canada

\section{Letourneau-Guillon, MD, MSc, FRCPC}

Department of Radiology, Centre Hospitalier de l'Université de Montréal (CHUM), Montreal, QC, Canada orientation of the needle bevel would thus represent a potentially important factor to investigate, since it is easy to modify.

In this prospective, randomized, blinded, cadaver study, we hypothesized that a caudal orientation of the Tuohy needle bevel should orient the catheter towards the ventral pleuro-vertebral part of the paravertebral space and would facilitate catheter insertion by diverting it away from the osseous components of the paravertebral space. Following ethics approval from the Centre Hospitalier de l'Université de Montréal, continuous paravertebral thoracic blocks were performed with $18 \mathrm{G}$ Tuohy needles and multi-orifice catheters (B-Braun Contiplex, B-Braun Medical Inc., Bethlehem, PA, USA) on ten cadavers by an experienced anesthesiologist, using a parasagittal, in-plane, cephaladto-caudad, ultrasound-guided approach at the T6 level. The primary endpoint of our study was the ease of insertion of the catheters, graded using a standardized scale as shown in the Figure.

For the initial insertion attempt, one side of the cadaver was randomized to a caudal (bevel down) orientation and the other side to a cephalad (bevel up) orientation. After confirmation of the needle tip position in the paravertebral space and injection of $5 \mathrm{~mL} 0.9 \%$ saline solution to distend the space, another investigator (blinded to the orientation allocation) attempted to insert the catheter and graded the ease of insertion using the standardized scale. In case of a failed attempt, injection of additional aliquots of $5 \mathrm{~mL}$ saline was allowed at one and three minutes. If catheter insertion remained impossible after five minutes, the needle was rotated $180^{\circ}$. Once insertion was successful, the catheter was advanced $3 \mathrm{~cm}$ and secured. Radiocontrast was then injected through each catheter and the extent of the solution spread was determined radiographically. 
A

\begin{tabular}{|l|l|}
\hline Gradation & Description of the ease of catheter insertion \\
\hline 1 & Very easy, successful at the first attempt \\
\hline 2 & Easy, successful in less than 1 minute \\
\hline 3 & Moderate, successful in more than 1minute but less than 3 minutes \\
\hline 4 & Difficult, successful in more than 3 minutes but less than 5 minutes \\
\hline 5 & Impossible after 5 minutes \\
\hline
\end{tabular}

B

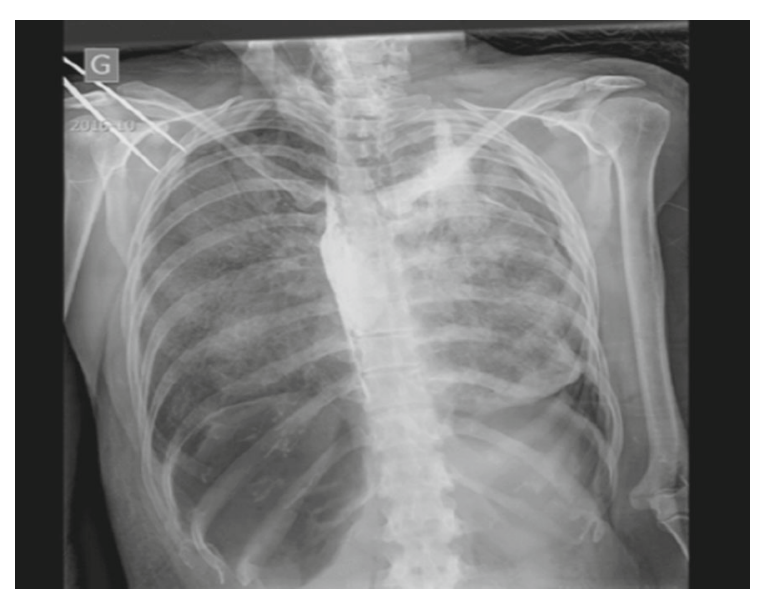

Figure (A) Standardized scale to describe the ease of catheter insertion. (B) Chest $x$-ray showing an adequate spread of the contrast media.

Twenty paravertebral punctures (each side of ten cadavers) were evaluated. The median [interquartile range (IQR)] ease of insertion was 2.5 [1-3] in the cephalad group and $2.0[1-3]$ in the caudal group (median difference, $-0.5 ; 95 \%$ confidence interval $[\mathrm{CI}],-2.0$ to $1.0 ; P=0.93)$. The median [IQR] time of insertion was 68 [13-93] sec and 30 [13-111] sec in the bevel up and bevel down groups, respectively (median difference, $-38 ; 95 \%$ CI, -164.1 to $29.0 ; P=0.85)$. Five catheters $(25 \%)$ were impossible to insert in their original allocation group, three in the cephalad group and two in the caudal group. After a $180^{\circ}$ rotation of the needle, four out the five catheters $(80 \%)$ were successfully inserted, with a median [IQR] score of ease of insertion of 1.7 [1-3]. One catheter remained impossible to insert beyond $3 \mathrm{~cm}$ despite rotation of the bevel. It was secured at the tip of the needle to be included in the contrast media distribution analysis. After the injection of the contrast media via the catheters, paravertebral diffusion was deemed adequate in $22.2 \%$ (2/ 9) and $90.9 \%(10 / 11)$ for the cephalad and caudal groups, respectively $(P=0.045)$. If a bevel reorientation was needed because initial insertion was impossible, the final position of the bevel was considered for analysis.

In conclusion, needle bevel orientation does not seem to influence the ease of paravertebral catheter insertion when the block is performed in a parasagittal in-plane ultrasound-guided technique in human cadaver models. Nevertheless, a caudal orientation could improve injectate distribution. These results need confirmation in clinical practice, as properties of living human tissues could be different from cadavers. Further studies are needed to determine which approach provide the best success rate.
This work was presented at the 2018 SFAR (Société Française d'Anesthésie et de Réanimation) meeting in Paris on September 27 28th.

Conflicts of interest None declared.

Editorial responsibility This submission was handled by Dr. Hilary P. Grocott, Editor-in-Chief, Canadian Journal of Anesthesia.

Funding None.

\section{References}

1. Gilbert J, Hultman J. Thoracic paravertebral block: a method of pain control. Acta Anaesthesiol Scand 1989; 33: 142-5.

2. Scarfe AJ, Schuhmann-Hingel S, Duncan JK, Ma N, Atukorale YN, Cameron $A L$. Continuous paravertebral block for postcardiothoracic surgery analgesia: a systematic review and metaanalysis. Eur J Cardiothorac Surg 2016; 50: 1010-8.

3. Luyet $C$, Eichenberger $U$, Greif $R$, Vogt A, Szucs Farkas Z, Moriggl $B$. Ultrasound-guided paravertebral puncture and placement of catheters in human cadavers: an imaging study. $\mathrm{Br}$ J Anaesth 2009; 102: 534-9.

4. Luyet $C$, Herrmann $G$, Ross $S$, et al. Ultrasound-guided thoracic paravertebral puncture and placement of catheters in human cadavers: where do catheters go? Br J Anaesth 2011; 106: 246-54.

5. Krediet AC, Moayeri N, van Geffen GJ, et al. Different approaches to ultrasound-guided thoracic paravertebral block: an illustrated review. Anesthesiology 2015; 123: 459-74.

Publisher's Note Springer Nature remains neutral with regard to jurisdictional claims in published maps and institutional affiliations. 